\title{
¿Existe Violencia Escolar en Educación Infantil y Primaria? Una propuesta para su evaluación y gestión
}

\author{
Natalia Albaladejo-Blázquez*, Rosario Ferrer-Cascales, Abilio Reig-Ferrer y Má-Dolores Fernández-Pascual
}

Departamento de Psicología de la Salud. Universidad de Alicante

\begin{abstract}
Resumen: La tradición investigadora de la violencia escolar se ha centrado fundamentalmente en los últimos ciclos de Educación Primaria y en la Educación Secundaria Obligatoria, abordándose muy poco la presencia, características, evaluación e intervención de los problemas interpersonales en edades tempranas. El objetivo del presente estudio es la construcción y validación de un cuestionario que identifique el inicio y las formas más frecuentes de violencia escolar en los primeros años de escolarización para diseñar programas de prevención eficaces. Los participantes fueron $195 \mathrm{ni}$ ños/as del último curso de educación infantil y primer ciclo de educación primaria. La investigación realizada ha mostrado que el instrumento denominado Cuestionario de Evaluación de Violencia Escolar en Infantil y Primaria (CEVEIP) presenta adecuadas propiedades psicométricas. El instrumento consta de 27 ítems en formato de autoinforme que evalúa siete tipologías de violencia que se dan en el contexto escolar en edades tempranas desde tres perspectivas: violencia observada, vivida y realizada. Los resultados ponen de manifiesto que el comportamiento violento está presente desde los primeros años de escolarización, siendo éste de baja o moderada frecuencia e intensidad. Estos niveles de violencia podrían ser aprovechados en el ámbito educativo para una gestión más positiva en el desarrollo personal del alumno/a.

Palabras clave: violencia escolar; infantil; primaria; evaluación; propiedades psicométricas.
\end{abstract}

Title: Does School Violence Occur in Preschool and Primary Education? An Assessment and Management Proposal.

Abstract: Traditional research on school violence has focused mainly on the later primary education grades and on secondary education, and has barely addressed the presence, characteristics, assessment, and intervention of interpersonal problems at early ages. The aim of this study was to construct and validate a questionnaire that identifies the onset and the most frequent forms of school violence in the first years of schooling in order to design effective prevention programs. The study participants were 195 children from the last year of preschool and the first stage of primary education. The research carried out demonstrates that the instrument, the Questionnaire for the Assessment of School Violence in the Preschool and Primary School (Cuestionario de Evaluación de Violencia Escolar en Infantil y Primaria, CEVEIP), has adequate psychometric properties. The instrument includes 27 self-report items that assess seven typologies of violence that occur in the school setting at early ages from three perspectives: observed violence, experienced violence, and perpetrated violence. The results reveal that violent behaviour of a low or moderate frequency and intensity is present, beginning in the first years of schooling. These levels of violence could be better managed in the educational setting in order to enhance students' personal development.

Keywords: school violence, preschool, primary school, assessment, psychometric properties.

\section{Introducción}

Los centros escolares son espacios de formación integral, interacción y aprendizaje para el desarrollo personal. El marco educativo es el contexto idóneo para el aprendizaje de contenidos curriculares y para que se produzcan los procesos relacionales, pero es en este espacio donde también surgen conflictos interpersonales. El conflicto puede ser definido como una situación de confrontación de dos o más personas, entre las cuales existe un antagonismo motivado por una confrontación de intereses (Defensor del Menor de la Comunidad de Madrid, 2006; Fernández, 1999; Monclús, Sánchez, Gonzalo y Barrigüete, 2004). No obstante, el conflicto en sí no tiene que cursar con agresividad, aunque sea difícil eludir cierto grado posiblemente inherente al mismo (Ortega, Romera, Mérida y Monks, 2009), pero el conflicto mal gestionado sí que puede derivar en problemas de convivencia en los centros educativos, dando lugar al fenómeno de la violencia escolar.

Entendemos por violencia escolar cuando una persona es agredida físicamente, insultada, socialmente excluida, amenazada o atemorizada por otros/as de forma puntual y no reiterada (Ortega, Del Rey y Mora-Merchán, 2001), caracterizándose por ser una acción intencional deliberadamente

* Dirección para correspondencia [Correspondence address]: Natalia Albaladejo-Blázquez. Departamento de Psicología de la Salud. Facultad de Ciencias de la Salud. Universidad de Alicante, Alicante. E-mail: natalia.albaladejo@,ua.es encauzada a provocar daño de baja o moderada intensidad, la cuál se lleva a cabo en contextos escolares, ya sea dirigida al alumnado, al profesorado o a los objetos propios del entorno educativo.

La violencia en el contexto escolar es un fenómeno general que se produce en todos los países en los que se ha estudiado y afecta a todas las clases sociales. En las últimas décadas se han incrementado las investigaciones tanto a nivel nacional como internacional, centrándose fundamentalmente en los últimos ciclos de educación primaria y en educación secundaria. Los primeros estudios en edades tempranas de escolarización se realizaron hace menos de dos décadas, y todos ellos informan que este tipo de conductas se encuentran presentes a estas edades. Así, la violencia escolar se ha observado, por ejemplo, en guarderías noruegas (Alsaker y Nagele, 2008; Alsaker y Vilén, 2010), en jardines de infancia en Estados Unidos (Kochenderfer y Ladd, 1996), en Suiza (Alsaker y Valkanover, 2001; Perren y Alsaker, 2006), así como en el Reino Unido, Italia y España (Monks, Palermiti, Ortega y Costabile, 2011; Ortega y Monks, 2005). No obstante, es necesario desarrollar investigaciones que nos permitan un mayor conocimiento de las características y topografía de la violencia escolar en edades tempranas (Alsaker y Vilén, 2010).

En la actualidad existen pocos estudios que describan la prevalencia, características, evaluación e intervención a través de la prevención de la violencia escolar en los primeros años de escolarización debido en gran parte a las dificultades metodológicas para valorar este tipo de conductas en 
edades tempranas (Caruana, 2011). En la literatura científica encontramos que se han aplicado técnicas de observación (Alsaker y Nagele, 2008; Alsaker y Valkanover, 2001; Collel y Escudé, 2006), técnicas de entrevistas o autoinformes cumplimentados por los adultos, fundamentalmente por padres o docentes (Alsaker y Valkanover, 2001; Monks et al., 2011), siendo los más utilizados en estos grupos de edad los informes cumplimentados por los propios niños, denominados nominación entre iguales a través de estudios sociométricos. Revisiones de la literatura científica muestran que la sociometría, como método de evaluación, presenta serias dificultades para que la nominación de preferencia positiva o negativa (sociometría ordinaria) sea interpretable en la dinámica de los roles de la violencia, ya que es un fenómeno con muchas connotaciones emocionales (Ortega y Monks, 2005; Ortega, Sánchez y Menesini, 2002).

Frente a la sociometría, los autoinformes, cuestionarios y escalas han demostrado ser una de las técnicas más apropiadas para la recogida de información en este tipo de estudios. $\mathrm{El}$ autoinforme permite evaluar aspectos cognitivos y emocionales, obtener información con una inversión mínima de tiempo y hallar puntuaciones que facilitan la interpretación de los resultados, presentando garantías métricas apropiadas. Estas pruebas son las que más se utilizan en la práctica diaria en el ámbito escolar (Muñiz y Fernández-Hermida, 2010).

Actualmente disponemos de un importante número de instrumentos que evalúan el acoso escolar (véase Calderero, Salazar y Caballo, 2011). El instrumento más difundido y empleado para evaluar el acoso entre iguales de alta o elevada intensidad es el Olweus Bully/Victim Questionnaire $(O B / V Q)$, cuya primera versión surge en 1986 (Olweus, 2010) y a partir de entonces se han publicado diversas revisiones y adaptaciones que se han aplicado en diferentes países (Caballo, Calderero, Arias, Salazar e Irurtia, 2012). A nivel internacional encontramos instrumentos centrados en evaluar las agresiones interpersonales y problemas de convivencia escolar desde el marco teórico del concepto de violencia escolar, destacando el Teacher Rating Instrument, TRI (Dodge y Coie, 1987), el Reactive-Proactive Agression Questionnaire, RPQ (Raine et al., 2006) o el Aggression Questionnaire, $A Q$ (Buss y Perry, 1992). A nivel nacional disponemos de cuestionarios que evalúan la violencia entre iguales junto a otros problemas de convivencia, como el "Cuestionario sobre los Problemas de la Convivencia Escolar, CPCE" (Peralta, Sánchez, Trianes y De la Fuente, 2003), el "Cuestionario para la Evaluación de la Violencia entre iguales en la Escuela y en el Ocio, CEVEO” (Díaz-Aguado, Martínez y Martín, 2004), el test “Acoso y Violencia Escolar, AVE” (Piñuel y Oñate, 2006), el "Cuestionario de Violencia EscolarRevisado, CUVE-R” (Álvarez-García, Núñez, Rodríguez, Álvarez y Dobarro, 2011), el "Cuestionario de Violencia Escolar Cotidiana, CUVECO” (Fernández-Baena, Trianes, Morena-Fernández, Escobar-Espejo, Infante-Cañete y Blanca-Mena, 2011) o el "Cuestionario Multimodal de Interacción Escolar, CMIE-IV" (Caballo et al., 2012) entre otros. Todos los instrumentos citados anteriormente van dirigidos a alumnado a partir de segundo ciclo de primaria, y sobre todo a adolescentes. Sin embargo, la edad es una variable importante a tener en cuenta ya que diferentes investigaciones llevadas a cabo sobre este tema evidencian que la detección precoz tiene una función importante en la prevención de este tipo de situaciones (Alsaker y Vilén, 2010; Ortega y Monks, 2005).

En este sentido, y tras una revisión sistemática de la literatura científica, no encontramos ningún autoinforme con criterios métricos de calidad que nos permitiera evaluar la violencia escolar en los primeros años de escolarización, por lo que nuestro grupo decidió elaborar un cuestionario para evaluar la presencia de violencia escolar en estos grupos de edad e investigar acerca del comportamiento y las relaciones entre los niños/as en estas primeras etapas de escolarización, determinando la naturaleza exploratoria y descriptiva del fenómeno de la violencia escolar de intensidad baja o moderada entre iguales, como experiencia de validación.

La presente investigación se ha llevado a cabo en colaboración con el Centro de Formación, Innovación y Recursos Educativos (CEFIRE) de Elda (Alicante), organismo dependiente de la Consejería de Educación, Formación y Empleo de la Generalitat Valenciana. Este Centro ha puesto en marcha diversas iniciativas para mejorar la convivencia en los centros y prevenir la violencia escolar en adolescentes (Caruana, 2005, 2007). Con este estudio se ha iniciado una línea de trabajo con la finalidad de prevenir la violencia y potenciar la convivencia escolar en educación infantil y primaria.

El objetivo general de este trabajo es conocer la presencia e inicio de la violencia escolar entre iguales, protagonizadas por los alumnos/as en edades tempranas de escolarización, y las distintas tipologías que se dan en los centros educativos. Para ello, se ha desarrollado y validado un nuevo instrumento, estandarizado y autoadministrado, denominado "Cuestionario de Evaluación de Violencia Escolar en Infantil y Primaria" (CEVEIP). Este instrumento deberá contar con garantías métricas adecuadas y ser útil para su aplicación en el ámbito educativo.

\section{Método}

\section{Participantes}

Han participado un total de 195 estudiantes, de centros educativos concertados-privados de educación infantil y educación primaria de la localidad de Elda, provincia de Alicante. Participó el total del alumnado matriculado en el último curso de educación infantil (100); y la mitad (95) de los alumnos/as matriculados en los dos cursos que comprenden el primer ciclo de educación primaria de dos centros educativos (47 de primer curso y 48 de segundo curso). Para la distribución del alumnado se respetó la formación natural de los grupos por clases. Con respecto al género, el 59.5\% de los alumnos son niños (30.3\% de educación infantil y $29.2 \%$ de educación primaria) y el $40.5 \%$ son niñas $(21 \%$ de educación infantil y $19.5 \%$ de educación primaria). Las edades 
están comprendidas entre 5 y 8 años $(M=6.08 ; D T=1 ; M o$ $d a=6$; Mediana $=6$ ). Para las niñas, la media de edad es de 6.04 años $(D T=0.88)$ y para los niños la media es de 6.10 años $(D T=1.07)$. No se han encontrado diferencias estadísticamente significativas con respecto al género $\left(\chi^{2}(3 \mathrm{~g} .1)=\right.$..02 , $p=.88)$, ni la edad $(t=.46, p=.65)$.

\section{Diseño}

Se emplea un diseño instrumental de desarrollo y adaptación de cuestionarios de medida con diseño de investigación de tipo descriptivo y transversal.

\section{Instrumento}

"Cuestionario de Evaluación de Violencia Escolar en Infantil y Primaria" (CEVEIP). Este instrumento ha sido elaborado ad hoc con el objetivo de conocer la presencia e inicio de la violencia escolar entre iguales protagonizada por los alumnos/as en edades tempranas de escolarización. Para ello se partió de una exhaustiva búsqueda bibliográfica de la literatura científica en las principales bases de datos (Psicodoc, CSIC-ISOC, Eric, Teseo, PsycINFO, Scopus, Medline), así como una revisión manual de las principales revistas especializadas, monografías y compilaciones, tanto de ámbito nacional como internacional, sobre la evaluación de la violencia escolar en la que localizamos un total de 26 autoinformes dirigidos a pre-adolescentes y adolescentes. Las palabras clave utilizadas en la búsqueda fueron: violencia entre iguales, violencia escolar, maltrato entre iguales, bullying, evaluación, propiedades psicométricas, convivencia, víctima, agresor, espectador, prevención violencia, infantil, primaria.

Se diseñó el plan de trabajo comenzando por la revisión de los distintos instrumentos, seguido de un estudio Delphi con participación de un total de 10 jueces expertos en la elaboración de instrumentos de medida y profesionales del ámbito de la educación con experiencia profesional que desempeñan funciones en los niveles educativos objeto de nuestro estudio, para establecer un marco teórico de referencia y un consenso sobre el contenido y estructura preliminar que debería tener el instrumento. Para el desarrollo del instrumento se utilizó como marco teórico la definición de violencia escolar anteriormente comentada, tomando como modelo y punto de partida el cuestionario elaborado por el estudio del Defensor del Pueblo $(1999,2006)$ y la investigación de Díaz-Aguado et al. (2004) con población adolescente.

Posteriormente, se procedió a seleccionar, por parte de cada uno de los miembros del equipo de trabajo, aquellos ítems considerados importantes para evaluar situaciones de violencia escolar en estas edades. A continuación se discutieron uno a uno los ítems propuestos en sesión plenaria, a través de la técnica focus group (Morgan, 1998a; 1998b). Finalizada la valoración de los ítems, atendiendo a la corrección sintáctica, claridad semántica y la adecuación para la población a quien va dirigido el instrumento, se obtuvo la versión piloto que conformó el instrumento inicial.
La versión piloto fue administrada a un total de 15 alumnos/as de educación infantil y 25 de educación primaria (10 alumnos/as del primer curso y 15 del segundo curso) para valorar su comprensión y factibilidad de administración. A partir de los datos obtenidos, los miembros del equipo de investigación revisaron la versión piloto del cuestionario y realizaron una serie de propuestas de modificación, obteniéndose de esta forma la versión definitiva del instrumento.

La versión definitiva del CEVEIP quedó conformada por un total de 30 ítems conceptualmente adecuados para evaluar los tipos de violencia escolar entre iguales en edades tempranas de escolarización y su frecuencia en tres situaciones de violencia o victimización: actos presenciados, vividos o realizados. De la totalidad de los ítems, se seleccionaron un total de 22 de los cuestionarios originales que sirvieron de base para la elaboración del instrumento, los cuáles fueron reformulados para poder ser aplicados en niños/as de educación infantil y primaria. Además se incorporaron 8 ítems nuevos elaborados por el grupo de profesionales y expertos que incluían situaciones no contempladas en estos cuestionarios.

El autoinforme se complementa con seis preguntas criteriales de interés: cuando a ti o a un compañero le sucede alguno de los problemas mencionados en el apartado anterior, ¿se lo dices a la familia?, ¿'se lo dices al profesor/a?, ¿te vas sin decir nada? Cada una de las preguntas tiene una formulación doble: "a ti" y "a un compañero/a", haciendo referencia a situaciones vividas por el escolar que responde y situaciones que presencia en los demás. Tanto las respuestas a cada uno de los ítems del CEVEIP como a las variables criteriales se presentan en formato tipo Likert con cuatro opciones de respuesta (1=nunca, $2=$ pocas veces, $3=$ muchas veces y $4=$ siempre).

En el instrumento también se incluyeron variables sociodemográficas de interés para nuestro estudio como la edad, el género y el ciclo educativo.

\section{Procedimiento}

En primer lugar se solicitó la autorización de la dirección de los centros escolares y el consentimiento informado de los padres/madres y/o tutores de los niños/as y se les informó de las características del estudio, asegurándoles tanto el anonimato como la confidencialidad de los resultados y la voluntariedad de participar en el mismo. El pase del instrumento se llevó a cabo por dos psicopedagogos/as que habían participado en la elaboración del instrumento y previamente fueron entrenados a tal efecto. Las pruebas se administraron en horario lectivo a los alumnos/as.

En el caso de los alumnos/as de infantil, la aplicación del cuestionario se llevó a cabo en grupos de dos alumnos/as fuera del aula. Se leían las preguntas e indicaban las diferentes opciones de respuesta, siendo el alumno/a quien elegía la opción de respuesta pegando un gomet en la casilla escogida. En el caso de los alumnos/as de primaria, el aula se dividió en dos para que los grupos fueran menos numerosos, se le- 
ían las preguntas y las opciones de respuesta a las mismas y el alumno/a colocaba una cruz en la casilla de la opción de la respuesta escogida. A la hora de recoger los cuestionarios se verificaban si estaban cumplimentados correctamente.

\section{Análisis de datos}

En primer lugar se llevó a cabo un análisis descriptivo de carácter exploratorio basado en una estadística univariable de las variables de interés. Para explorar la presencia o ausencia de violencia escolar en edades tempranas, utilizamos un análisis de agrupación de los ítems en dos categorías: valores altos y bajos. Como medida de comparación de grupos se ha empleado la prueba de contraste de hipótesis $\chi^{2}$. En segundo lugar, con el objeto de evaluar la estructura del cuestionario a partir de las puntuaciones de los ítems que lo componen, se llevaron a cabo análisis factoriales exploratorios para cada una de las escalas de situaciones de violencia escolar (presenciadas, vividas, realizadas), con extracción de componentes principales y posterior rotación oblicuo (PROMAX). En este sentido se han utilizado matrices de correlación policórica dado que está especialmente indicada en los casos en los que los ítems presentan un formato de respuesta tipo Likert (Muthen y Kaplan, 1992). A partir de los factores obtenidos en el primer análisis factorial, se llevó a cabo un segundo nivel de análisis con el objetivo de lograr una mayor reducción de variables, pasando a continuación a calcular la consistencia interna de cada uno de los factores a través del coeficiente alfa de Cronbach. Una vez realizado el análisis factorial y la consistencia interna, se transformaron las puntuaciones directas en puntuaciones transformadas en una escala de 0 a 10 para una mejor interpretabilidad. Por último, se realizaron análisis para estudiar estos factores valorando las diferencias según las variables género y ciclo educativo y como medida de comparación de grupos se empleó la prueba $t$ Student. Los análisis de los datos se han realizado con el programa estadístico SPSS., v.18.0 para Windows, (SPSS Inc, Chicago, USA) y el programa FACTOR 7.2. (Lorenzo-Seva y Ferrando, 2006) para el análisis factorial exploratorio, debido a la posibilidad que ofrece, por un lado, de realizar análisis utilizando matrices de correlación policórica y, por otro, proporcionar el análisis paralelo.

\section{Resultados}

Al objeto de explorar la presencia o ausencia de violencia escolar, se presenta la relación abreviada de los ítems del CEVEIP (ver tabla 1), con los resultados relativos al sumatorio de los porcentajes, agrupados en dos amplias categorías de alternativas de respuesta, denominadas de valor alto (dos categorías de alta frecuencia: siempre y mucha veces) y de valor bajo (dos categorías de baja frecuencia: nunca y pocas veces). También se analizó, a través de la prueba de contraste de hipótesis $\chi^{2}$, las diferencias en función del género y el ciclo educativo (infantil o primaria) en la que se encuentra el alumnado.
Tabla 1. Relación abreviada de los ítems para cada una de las escalas y análisis bivariado por género y ciclo educativo del CEVEIP.

\begin{tabular}{|c|c|c|c|c|c|c|}
\hline \multirow[t]{2}{*}{ CEVEIP } & \multicolumn{4}{|c|}{$\begin{array}{l}\text { Valor Valor Género } \\
\text { Baio Alto }\end{array}$} & \multicolumn{2}{|c|}{$\begin{array}{c}\text { Ciclo } \\
\text { educativo }\end{array}$} \\
\hline & $\begin{array}{c}\text { Bajo } \\
(\%)\end{array}$ & $\begin{array}{l}\text { Alto } \\
(\%) \\
\end{array}$ & \multicolumn{2}{|c|}{$\chi^{2} \quad p$} & \multirow[t]{2}{*}{$\chi^{2}$} & \multirow[t]{2}{*}{$p$} \\
\hline ones Pre & & & & & & \\
\hline 1. In & 81 & 19 & 3.27 & .35 & 28.45 & .00 \\
\hline 2. In & 63.3 & 36.4 & 3.43 & .32 & 14.60 & .00 \\
\hline 3. $\mathrm{Pe}$ & 75.4 & 24.6 & 1.88 & .59 & 49.56 & .00 \\
\hline 4. $\mathrm{P}$ & 59 & 41 & 2.83 & .41 & 14.31 & .00 \\
\hline 5. E & 67.7 & 32.3 & 4.63 & .20 & 32.60 & .00 \\
\hline & 75.9 & 24.1 & 9.41 & .02 & 10.21 & .01 \\
\hline 7. Q $Q$ & 83 & 17 & 1.10 & .77 & 26.58 & .00 \\
\hline 8. E & 89.2 & 10.8 & 1.02 & .79 & 13.56 & .00 \\
\hline \multicolumn{7}{|l|}{ Situaciones Vividas } \\
\hline 9 Insultar & 9.3 & 10.7 & 2.21 & .52 & 16.42 & \\
\hline & 3.3 & 16.7 & 5.68 & .12 & 5.76 & .12 \\
\hline & 6.7 & 13.3 & .37 & .94 & 19.46 & \\
\hline & & 18 & 7.43 & .05 & 2.05 & \\
\hline & & 12.6 & 4.14 & .24 & 5.62 & . \\
\hline & 2 & 12.8 & 3.63 & .30 & 2.52 & .47 \\
\hline & 8.2 & 11.8 & 3.12 & .37 & 6.49 & .09 \\
\hline & 3 & 9.7 & 9.87 & .02 & 7.45 & .05 \\
\hline & 5 & 79.5 & 1.86 & .60 & 11.28 & .01 \\
\hline & 8.8 & 11.2 & 1.87 & .60 & 2.57 & .46 \\
\hline & 9.7 & 10.2 & 5.74 & .12 & 9.43 & .02 \\
\hline $\begin{array}{l}\text { 20. Me de } \\
\text { doy o has }\end{array}$ & 0.2 & 9.8 & 2.15 & .54 & 2.28 & \\
\hline \multicolumn{7}{|l|}{ Situaciones Realizadas } \\
\hline & & 2 & 3.38 & .33 & 18.15 & .00 \\
\hline & 4 & 5.6 & 1.06 & .58 & 7.28 & .02 \\
\hline & & 1 & 8.24 & .04 & 16.84 & .00 \\
\hline & 5 & 4.6 & 4.72 & .09 & 6.71 & .03 \\
\hline & 7 & 2.6 & 5.22 & .15 & 4.45 & .21 \\
\hline & 96.9 & 3.1 & 6.42 & .04 & 7.88 & .01 \\
\hline & 99.5 & 0.5 & 4.62 & .09 & 1.42 & .49 \\
\hline 28. E & 99 & 1 & 6.44 & .09 & 8.13 & .04 \\
\hline 29. No dejar jugar $\mathrm{c}$ & 72.8 & 27.2 & 1.19 & .75 & 12.61 & .00 \\
\hline 30. Si quiero conseguir les pid & 91.8 & 8.2 & 1.44 & .69 & 8.12 & \\
\hline
\end{tabular}
$\begin{array}{lllllll}\text { 30. Si quiero conseguir les pido } \quad 91.8 & 8.2 & 1.44 & .69 & 8.12 & .04\end{array}$ Nota: Valor Alto=agrupación de opciones de respuesta "siempre y muchas veces"; Valor Bajo=agrupación de opciones de respuesta "nunca y pocas veces".

Los resultados nos indican que 116 alumnos/as, nunca o pocas veces, han presenciado, vivido o realizado situaciones de violencia escolar. Predomina la presencia de violencia física y verbal directa que se realiza fuera del aula (pegar e insultar en el recreo), dándose más desde la perspectiva del espectador que desde la de la víctima o agresor. Hay que destacar que, desde la perspectiva del agresor, los escolares manifiestan los porcentajes más elevados en las situaciones de violencia relacional (no dejar jugar o pedir algo a cambio).

En cuanto a las diferencias entre chicos y chicas, se percibe que ellos están más implicados que ellas en las formas directas de agresión y de victimización, cabe destacar la diferencia estadísticamente significativa en el ítem "pegar en clase" en las situaciones realizadas, siendo los chicos quienes manifiestan dicho comportamiento en mayor medida que las chicas. Por otro lado, encontramos dos diferencias estadísticamente significativas a favor de las chicas, en la situación 
presenciada y realizada "haber molestado o no dejar hacer el trabajo".

En relación al ciclo educativo, se observa que las diferentes manifestaciones de violencia escolar se han identificado en las dos etapas educativas (infantil y primaria). A pesar de ello, son los alumnos/as del último curso de educación infantil quienes manifiestan en mayor medida haber presenciado, vivido y realizado las modalidades de violencia física, verbal y relacional, tanto dentro como fuera del aula; a excepción de la situación presenciada "quitar o esconder las cosas", las situaciones vividas "dejar jugar" y "jugar solo en el recreo" y las situaciones realizadas "molestar o no dejar trabajar" y "estropear el trabajo", que nos revelan que son los escolares de primaria quienes las indican en mayor proporción que los de infantil.

Referente a las respuestas señaladas por los escolares en las preguntas criteriales que indican cuando los alumnos/as se dirigen a los adultos en busca de ayuda en situaciones de violencia escolar, o si por el contrario, se van sin decir nada; los resultados nos indican que existe un bajo porcentaje de alumnado que se va sin decir nada a nadie cuando tienen un problema o cuando percibe que alguien puede tenerlo. Hay que destacar que en el análisis de diferencias se observa que los escolares de infantil cuando tienen un problema $\left(\chi^{2}\right.$ $(3 \mathrm{~g} .1)=22.88,. p<.00)$, o cuando ven que alguien puede tenerlo $\left(\chi^{2}\right.$ (3g.l.) $\left.=10.74, p<.05\right)$, acuden a comunicárselo al profesorado en mayor proporción que a la familia. No se encuentran diferencias estadísticamente significativas con respecto al género.

A continuación, se realizó el análisis de discriminación de los 30 ítems, eliminando aquellas correlaciones ítem-total corregidas inferiores a .30 , ya que se considera que discriminan muy pobremente (Nunnally y Bernstein, 1995). Tras este análisis se detectó que todos los ítems cumplían los criterios propuestos a excepción de 6 ítems. No obstante, se acordó mantener tres ítems $(18,19$ y 20$)$ por considerarse de interés la evaluación de su contenido (violencia física y exclusión social). Se decidió eliminar los ítems 17, 29 y 30 porque mejoraba sensiblemente la fiabilidad del cuestionario. Así, el cuestionario quedó reducido a 27 ítems con una elevada consistencia interna (alfa de Cronbach $=.86$ ), no encontrándose ningún ítem cuya eliminación aumentara la fiabilidad del CEVEIP global.

Tabla 2. Estructura factorial exploratoria del CEVEIP para las Situaciones de Violencia Escolar.

\begin{tabular}{|c|c|c|c|c|}
\hline Situaciones Presenciadas & Factor 1 & Factor 2 & & Correlación ítem-escala \\
\hline A8. Estropearle los trabajos & .84 & & & .63 \\
\hline A7. Quitarle o esconderle las cosas & .62 & & & .38 \\
\hline A6. Molestarle o no dejarle trabajar & .65 & & & .54 \\
\hline A3. Pegarle en clase & .63 & & & .60 \\
\hline A4. Pegarle en el recreo & .59 & & & .52 \\
\hline A1. Insultarle en clase & & .72 & & .45 \\
\hline A5. Empujarle o fastidiarle en la fila & & .44 & & .55 \\
\hline A2. Insultarle en el recreo & & .36 & & .47 \\
\hline Varianza Explicada (\%) & 47.63 & 13.86 & & \\
\hline Situaciones Vividas & Factor 1 & Factor 2 & Factor 3 & Correlación Ítem-escala \\
\hline A15. Me quitan o esconden las cosas & .63 & & & .53 \\
\hline A13. Me empujan o fastidian en las filas & .77 & & & .48 \\
\hline A18. No se quieren sentar conmigo & .44 & & & .11 \\
\hline A16. Me estropean los trabajos & .49 & & & .35 \\
\hline A9. Me insultan en clase & & .72 & & .37 \\
\hline A11. Me pegan en clase & & .77 & & .60 \\
\hline A10. Me insultan en el recreo & & .72 & & .39 \\
\hline A12. Me pegan en el recreo & & .48 & & .48 \\
\hline A19. Tengo que jugar solo en el recreo & & & .70 & .37 \\
\hline A20. Me dejan jugar si les doy o hago lo que me dicen & & & .48 & .32 \\
\hline A 14. Me molestan o no me dejan trabajar & & & .37 & .39 \\
\hline Varianza Explicada (\%) & 35.60 & 12.28 & 10.93 & \\
\hline Situaciones Realizadas & Factor 1 & Factor 2 & & Correlación ítem-escala \\
\hline A22. He insultado en el recreo & .88 & & & .59 \\
\hline A24. He pegado en el recreo & .61 & & & .54 \\
\hline A23. He pegado en clase & .64 & & & .56 \\
\hline A21. He insultado en clase & .47 & & & .62 \\
\hline A25. He empujado o fastidiado en las filas & .53 & .40 & & .63 \\
\hline A28. He estropeado el trabajo & & .89 & & .31 \\
\hline A27. He quitado o escondido las cosas & & .44 & & .30 \\
\hline A26. He molestado o no he dejado trabajar & & .45 & & .41 \\
\hline Varianza Explicada (\%) & 58.69 & 12.21 & & \\
\hline
\end{tabular}

Se llevó a cabo un análisis factorial exploratorio para cada una de las escalas de situaciones de violencia escolar (pre- senciadas, vividas, realizadas) sobre la matriz de correlaciones policóricas con el fin de determinar la estructura facto- 
rial del cuestionario. Debido a que no cumple los criterios de normalidad multivariable del análisis de asimetría y curtosis multivariada de Mardia (1970), se ha empleado el Unweighted Least Squares (ULS) como método de extracción. El método de rotación oblicuo empleado ha sido PROMAX.

Los índices de la prueba de Esferecidad de Barlett para las tres escalas $(p=.00)$ y de la prueba de Kaiser-Mayer-Olkin $(K M O=.80$ en las situaciones presenciadas, $K M O=.75$ en las situaciones vividas y $K M O=.77$ en las situaciones realizadas) nos indicaban la idoneidad de los datos para realizar los análisis factoriales para cada una de las escalas de situaciones de violencia escolar. Para confirmar el número de factores a considerar en cada escala, se aplicó el criterio scree-test (Cattell, 1966) y el análisis paralelo (Horn, 1965), ambos criterios recomendaron una solución bifactorial para la escala situaciones presenciadas y situaciones realizadas, mientras que para las situaciones vividas proponían una solución trifactorial.

En la Tabla 2 se presentan los factores, con los ítems abreviados, de cada una de las escalas de situaciones de violencia escolar (presenciadas, vividas, realizadas), correlación ítem-escala y el porcentaje de varianza explicado.

Únicamente encontramos una saturación mayor a .35 en dos factores en el ítem 25 de la escala de situaciones de violencia realizadas. Este ítem se puede caracterizar por ser una agresión física directa e indirecta al mismo tiempo, al poder ejercerla el sustituto del agresor o espectador activo, a pesar de ello, atendiendo a la carga factorial e interpretación decidimos incluirlo en el primer factor.

La escala de situaciones de violencia escolar presenciadas mostró una estructura compuesta de dos factores que explican el $61.49 \%$ de la varianza total, atendiendo al contenido, el primer factor se denominó "violencia física presenciada" y el segundo factor "violencia verbal presenciada". Las escala de situaciones de violencia escolar vividas mostró una estructura compuesta por tres factores que explican el $58.82 \%$ de la varianza total. Al primer factor se denominó "violencia indirecta vivida", al segundo factor "violencia directa vivida" y al tercero "violencia psicosocial vivida". El análisis factorial de la escala de situaciones de violencia escolar realizadas arroja dos factores que explican el 70.90\% de la varianza total, denominando al primer factor "violencia directa realizada" y al segundo "violencia indirecta realizada".

Con el fin de evaluar el ajuste de la solución se calculó la raíz media cuadrática residual, obteniendo un valor en las situaciones presenciadas de $R M S R=.045$, en las situaciones vividas de $R M S R=.043$ y en las situaciones realizadas de $R M S R=.056$. Estos resultados nos indican un buen ajuste de cada una de las escalas de situaciones de violencia escolar, dado que el límite superior para considerar un ajuste aceptable según el criterio de Kelley es de .0718 (Kelley, 1935). Los índices de bondad de ajuste GFI para las tres escalas (situaciones presenciadas, vividas y realizadas) es de $G F I=.99$, indicándonos un buen ajuste en la proporción de covariación entre las variables explicadas, según el criterio de referencia de índice de bondad de ajuste GFI 95 (Ruíz, Pardo y San Martín, 2010).

Para calcular la simplicidad de las soluciones factoriales obtenidas, se utilizó el índice de Bentler de simplicidad (1977) según el cual valores cercanos a 1 señalan una máxima simplicidad de la solución obtenida. En cada una de las tres escalas de situaciones de violencia escolar obtenemos una elevada simplicidad de la solución obtenida: $S=.95$ en las situaciones presenciadas, $S=.96$ en las situaciones vividas y $S=.97$ en las situaciones realizadas.

Por otro lado, encontramos que las relaciones entre los distintos factores de cada una de las escalas de situaciones de violencia escolar son, en general, elevadas. La correlación entre los dos factores de las situaciones presenciadas (violencia física y violencia verbal) es de $r=.52, p<.01$. Entre los tres factores de la escala de situaciones vividas, encontramos diferencias estadísticamente significativas en todas ellas $(p<.01)$, siendo la más elevada la correlación entre violencia directa y violencia indirecta $(r=.55)$. Por último, la relación entre los factores violencia escolar vivida directa e indirecta es positiva $(r=.56, p<.01)$.

Tras el primer análisis factorial exploratorio, se llevó a cabo un análisis factorial de segundo orden para explorar las relaciones sumarias entre las variables factoriales. Nuestro objetivo era investigar la estructura factorial a partir de las dimensiones de primer orden de cada una de las escalas de situaciones de violencia escolar evaluadas. Este análisis factorial de segundo orden identifica una estructura unidimensional en cada una de las escalas de situaciones de violencia escolar evaluadas. En las tres escalas de situaciones de violencia, tanto el scree-test (Cattell, 1966) y el análisis paralelo (Horn, 1965) señalan la unidimensionalidad de las escalas, siendo el autovalor correspondiente al segundo factor de cada una de las escalas de situaciones de violencia escolar claramente inferior a 1 , al igual que todos los ítems cargan de forma sustantiva en cada factor (presenciada, vivida o realizada) con valores superiores a .40. Esto nos confirma la estructura conceptual inicial, una estructura tri-factorial del cuestionario (presenciada, vivida y realizada).

El análisis de fiabilidad queda reflejado en la Tabla 3. Los resultados ponen de manifiesto que la consistencia interna en las escalas de situaciones de violencia escolar (presenciada, vivida o realizada) y en el CEVEIP global son aceptables y similares entre ellos, tanto en las submuestras por género como en ciclo educativo.

Tabla 3. Fiabilidad de la estructura conceptual del CEVEIP.

\begin{tabular}{lccccc}
\hline & \multicolumn{5}{c}{ Alfa de Cronbach } \\
\cline { 2 - 6 } & Niño Niña Infantil Primaria & Total \\
\hline Violencia Escolar Presenciada & .81 & .79 & .81 & .75 & .80 \\
Violencia Escolar Vivida & .71 & .70 & .75 & .73 & .72 \\
Violencia Escolar Realizada & .79 & .78 & .80 & .76 & .79 \\
\hline CEVEIP global & .86 & .85 & .86 & .84 & .86 \\
\hline
\end{tabular}

El CEVEIP arroja siete factores referentes a situaciones de violencia en escolares del último curso de educación infantil y primer ciclo de educación primaria. Además de esta 
estructura factorial empírica de primer orden, se pueden obtener tres puntuaciones globales de segundo orden: a) Situaciones de Violencia Escolar Presenciadas, mediante la suma de las puntuaciones obtenidas en los factores violencia física presenciada y violencia verbal presenciada; b) Situaciones de Violencia Escolar Vividas, mediante la suma de las puntuaciones obtenidas en los factores violencia indirecta, violencia directa y violencia psicosocial vivida, y c) Situaciones de Violencia Escolar Realizadas, mediante la suma de violencia directa e indirecta realizada. Tanto los factores como las escalas del CEVEIP se interpretan en la línea de que a mayor puntuación más violencia existe en el centro escolar.
En la Tabla 4 se presentan las medias, desviaciones estándar y puntuaciones normalizadas (sobre una escala de 0 a 10) para cada una de las escalas de situaciones de violencia escolar (presenciadas, vividas y realizadas) y los factores del CEVEIP. Las puntuaciones normalizadas posibilitan una fácil y sencilla interpretación de las medias resultantes. De este modo, se observa como los escolares de infantil y primaria obtienen unas medias bajas en violencia escolar. El rango de puntuaciones transformadas oscila entre .53 (violencia indirecta realizada) y 2.82 (violencia física presenciada).

Tabla 4. Análisis descriptivo de los factores de primer y segundo orden del CEVEIP.

\begin{tabular}{lccc}
\hline CEVEIP & $\mathrm{n}^{\circ}$ ítems & $M(D T)$ & Puntuación Transformada \\
\hline I. Violencia física presenciada & 5 & $9.24(2.75)$ & 2.82 \\
II. Violencia verbal presenciada & 3 & $6.32(2.19)$ & 2.76 \\
III. Violencia indirecta vivida & 4 & $6.32(2.27)$ & 1.93 \\
IV. Violencia directa vivida & 4 & $6.49(2.30)$ & 2.07 \\
V. Violencia psicosocial vivida & 3 & $4.53(1.70)$ & 1.70 \\
VI. Violencia directa realizada & 5 & $6.55(2.01)$ & .03 \\
VII. Violencia indirecta realizada & 3 & $3.48(.83)$ & .53 \\
\hline Escala Situaciones de Violencia Presenciada & 8 & $15.87(4.89)$ & 3.28 \\
Escala Situaciones de Violencia Vivida & 11 & $17.35(4.74)$ & 1.92 \\
Escala Situaciones de Violencia Realizada & 8 & $10.04(2.51)$ & .85 \\
\hline CEVEIP total & 27 & $43.27(9.91)$ & .86 \\
\hline
\end{tabular}

Se analiza, a continuación (ver Tabla 5), las diferencias por género y ciclo educativo de los factores empíricos del instrumento de medida. En cuanto al género, apenas encontramos diferencias estadísticamente significativas en las escalas y factores de las situaciones de violencia escolar; única- mente encontramos diferencias estadísticamente significativas en la escala Situaciones de Violencia Escolar Realizadas y el factor violencia indirecta realizada, siendo los niños quienes obtienen mayores puntuaciones con respecto a las niñas.

Tabla 5. Descriptivos y diferencias en función del género y ciclo educativo.

\begin{tabular}{|c|c|c|c|c|c|c|}
\hline & Niño & Niña & & Infantil & Primaria & \\
\hline & $M(D T)$ & $M(D T)$ & $t$ & $M(D T)$ & $M(D T)$ & $t$ \\
\hline I. Violencia física presenciada & $9.27(2.75)$ & $9.18(2.77)$ & .21 & $9.72 \pm 3.07$ & $8.73 \pm 2.28$ & $2.54 *$ \\
\hline II. Violencia verbal presenciada & $6.25(2.22)$ & $6.44(2.15)$ & -.60 & $6.98 \pm 1.99$ & $5.64 \pm 2.19$ & $4.44 * *$ \\
\hline III. Violencia indirecta vivida & $6.28(2.23)$ & $6.37(2.34)$ & .28 & $6.60 \pm 2.38$ & $6.03 \pm 2.12$ & 1.75 \\
\hline IV. Violencia directa vivida & $6.77(2.48)$ & $6.08(1.94)$ & -2.06 & $7.05 \pm 2.57$ & $5.91 \pm 1.81$ & $3.56^{* *}$ \\
\hline V. Violencia psicosocial vivida & $4.52(1.81)$ & $4.55(1.55)$ & .12 & $4.37 \pm 1.58$ & $4.71 \pm 1.81$ & -1.41 \\
\hline VI. Violencia directa realizada & $6.77(2.08)$ & $6.22(1.85)$ & -1.88 & $8.31 \pm 2.48$ & $7.31 \pm 1.87$ & $3.16^{*}$ \\
\hline VII. Violencia indirecta realizada & $3.58(.90)$ & $3.34(.71)$ & $2.10^{*}$ & $2.21 \pm .49$ & $2.22 \pm .54$ & -.14 \\
\hline Violencia Escolar Presenciada & $15.68(4.84)$ & $16.13(4.97)$ & -.62 & $17.41 \pm 5.07$ & $14.25 \pm 4.12$ & $.77 * *$ \\
\hline Violencia Escolar Vivida & $17.58(4.92)$ & $17.02(4.48)$ & .80 & $18.02 \pm 5.24$ & $16.66 \pm 4.07$ & $2.02 *$ \\
\hline Violencia Escolar Realizada & $10.36(2.60)$ & $9.56(2.31)$ & $2.2^{*}$ & $10.52 \pm 2.67$ & $9.53 \pm 2.23$ & $2.78 * *$ \\
\hline
\end{tabular}
${ }^{*} p<.05 ; * * p<.01$

En relación al ciclo educativo, los resultados obtenidos indican que existen diferencias estadísticamente significativas tanto en las Situaciones de Violencia Escolar Presenciadas, Vividas y Realizadas, como en los factores violencia física y verbal presenciada, violencia directa vivida y violencia directa realizada; siendo en todos los casos los escolares del último curso de educación infantil quienes presentan mayores niveles que los de primer ciclo de primaria.

\section{Discusión y Conclusiones}

Los resultados del presente estudio respecto a la percepción del alumnado sobre la presencia e inicio de la violencia escolar en educación infantil y primer ciclo de primaria en los centros escolares, nos indican que el perfil de violencia escolar en estas edades es bajo, encontrándose presentes las manifestaciones de violencia escolar en los centros educativos. Por lo que, la violencia en el ámbito escolar en estas edades es conocida y reconocida por los escolares y forma parte de 
la vida diaria de los centros educativos. Un número considerable de escolares se ven implicados en alguna ocasión en ellas como espectadores, víctimas o agresores, datos que concuerdan con estudios previos llevados a cabo sobre este tema (Alsaker y Vilén, 2010; Cerezo, 2006; Monks, Smith y Swettenham, 2005; Craig, Pepler y Atlas, 2000; Wolke, Woods, Schulz y Stanford, 2001); lo cual nos indica que la violencia puede ser una forma de resolución de conflictos, que puede llegar a producir consecuencias negativas en el desarrollo intelectual, social y emocional del niño/a. Estos datos coinciden con otros estudios realizados con alumnos/as de edades más avanzadas (Caballo, Arias, Calderero, Salazar e Irurtia, 2011; Caballo et al., 2012; Caruana, 2005, 2007; Fernández-Baena et al., 2011 y Piñero-Ruiz, 2010) donde se muestra que en el ámbito escolar los alumnos/as refieren niveles bajos de violencia, aunque muchos revelan que los han sufrido durante su escolarización en alguna ocasión.

No obstante, los niveles de violencia que los escolares de educación infantil y primaria muestran, están muy lejos de ser motivo de alarma social (Cerezo, 2009; Defensor del Pueblo, 1999, 2006; Ortega et al., 2009; Viera, Fernández y Quevedo, 1998; y Zabalza, 1999) y no tienen por qué dificultar la convivencia en los centros escolares. Consideramos que si partimos de los conflictos naturales que se dan en el ámbito escolar, y trabajamos con nuestros niños/as cómo resolverlos de la forma más creativa y eficaz posible, estaremos enseñando a los/as alumnos/as que el conflicto tratado de manera constructiva es necesario para el desarrollo integral del ser humano, sin llegar a derivar el mismo en un problema de violencia escolar.

Para comprender la violencia escolar que se da en las escuelas en los primeros años de escolarización, así como para desarrollar soluciones eficaces, es fundamental disponer de instrumentos con garantías métricas de calidad que además cuenten con una amplia aceptación, fácil manejo y poco tiempo de aplicación, y que nos permitan hacer tanto análisis adecuados de las situaciones, como elaborar programas eficaces de intervención dirigidos a la prevención de este problema. Su evaluación requiere de nuevos y variados instrumentos que midan los distintos aspectos del constructo (Caballo et al., 2012).

Una de las aportaciones del presente estudio frente a otros estudios realizados con estos grupos de edad (Alsaker y Valkanover, 2001; Alsaker y Vilén, 2010; Monks et al., 2011; Ortega y Monks, 2005), es el diseño de un autoinforme que permite conocer la opinión de los escolares sobre la frecuencia de aparición de diferentes tipologías de violencia en su entorno escolar. La perspectiva del alumnado resulta particularmente importante para analizar este fenómeno, ya que informa desde dentro del sistema, de las relaciones que ocurren en el aula y en el centro (Álvarez-García et al., 2011).

La investigación realizada permite disponer de un instrumento (CEVEIP) con una buena fiabilidad y validez. La estructura factorial del instrumento en población infantil, es consistente con el modelo inicial propuesto en diversos es- tudios con población adolescente (Defensor del Pueblo, 1999, 2006; Díaz-Aguado et al., 2004, Fernández-Baena et al., 2010), ya que el análisis factorial de segundo orden confirma la estructura conceptual teórica propuesta (observada, vivida y realizada). Aunque se observa que cada una de las situaciones de violencia escolar se explican como un único factor, encontramos que la escala Situaciones de Violencia Escolar Presenciada identifica dos factores (física y verbal), la escala Situaciones de Violencia Escolar Vividas identifica tres factores (directa, indirecta y psicosocial) y la escala Situaciones de Violencia Escolar Realizadas dos factores (directa e indirecta). Los índices alfa de Cronbach fueron adecuados en cada una de las situaciones evaluadas y en la puntuación total del cuestionario, lo cual demuestra la cohesión de los ítems de cada factor para evaluar componentes de la violencia escolar en edades tempranas de escolarización.

Respecto a las diferencias según el género y ciclo educativo, se observa que tanto los niños como las niñas refieren manifestaciones de violencia escolar por igual. Estos resultados coinciden con los estudios llevados a cabo por autores como Carney y Merrel (2001), Ortega y Monks (2005) y Ortega et al. (2009), quienes afirman que la tendencia tradicional de encontrar diferencias en el tipo de violencia según el género está cambiando en la actualidad, no apreciándose diferencias entre chicos y chicas. Referente al ciclo educativo, nuestros datos confirman que los/as niños/as pequeños son más proclives a las formas agresivas cara a cara (directa física o verbal) dándose poco entre ellos/as las formas de agresión social indirectas (Bjorkqvist, Lagerspetz y Kaukianen, 1992; Collel y Escudé, 2006; Crick, Ostrov, Burr, Cullerton-Sen, Jansen-Yeh y Ralston, 2006; Galen y Underwood, 1997; Monks et al., 2005; Ortega y Monks, 2005). Estos resultados sugieren que en los niveles educativos objeto de estudio, los/as niños/as utilizan formas de comportamiento más directas, irreflexivas y menos premeditadas.

Con respecto a los aspectos contextuales, los resultados obtenidos en nuestro trabajo van en consonancia con los estudios llevados a cabo sobre este tema, señalando que es el recreo el lugar escogido con mayor frecuencia por los/as alumnos/as como escenario de situaciones de violencia escolar (Carney y Merrell, 2001; Cerezo, 2006; Piñuel y Oñate, 2005; Wolke et al., 2001).

Encontramos que existe un elevado porcentaje de alumnos/as que acude a los adultos cuando tienen problemas, siendo los escolares de infantil quienes tienden a comunicarlo más al profesor que a las familias. Mientras que en cursos más avanzados, tal y como indica la literatura científica, los/as alumnos/s acuden más a la familia que a los profesores cuando tienen problemas de convivencia en los centros escolares (Baldry y Farrington, 2004; Defensor del Pueblo, 1999, 2006; Díaz-Aguado et al., 2004; Menesini, Codecasa, Benelli y Cowie, 2003).

En resumen, el CEVEIP es un instrumento con propiedades psicométricas adecuadas para evaluar la violencia escolar de moderada o baja intensidad en edades tempranas de escolarización. Se trata de un instrumento de autoinforme 
breve y de fácil manejo dirigido a evaluar la presencia o ausencia de violencia escolar en las interacciones en los entornos escolares, con el fin de poder actuar en la prevención del inicio de este problema a través del diseño de programas y actividades dirigidas a la mejora de las relaciones interpersonales en el centro escolar.

No obstante, no podemos obviar una serie de limitaciones inherentes al instrumento y a la propia investigación. En primer lugar, el CEVEIP se ha adaptado a partir de esta muestra y se precisa de otras muestras que nos permitirán revisar el instrumento para poder confirmar su validez factorial y demás garantías métricas de calidad. En segundo lugar,

\section{Referencias}

Alsaker, F. D. y Nagele, C. (2008). Bullying in kindergarten and prevention. In Craig, W., y Pepler, D. (Ed.), In International Perspective on Understanding and Addressing Bullying. Volume I (pp. 230-252). PREVNet: Kingston, Canada.

Alsaker, F. D. y Valkanover, S. (2001). Early diagnosis and prevention of victimization in kindergarten. In Juvonen J., Graham, S., (Ed.), Peer Harassment in School (pp. 175-195). New York, NY: Guilford Press.

Alsaker, F. D. y Vilén, U. L. (2010). El bullying en la edad preescolar. En Ortega, R. (Ed.), Agresividad injustificada, bullying y violencia escolar (pp. 129164). Madrid: Alianza Editorial.

Álvarez-García, D., Núñez, J. C., Rodríguez, C., Álvarez, L. y Dobarro, A. (2011). Propiedades psicométricas del Cuestionario de Violencia Escolar-Revisado (CUVE-R). Revista de Psicodidáctica, 16 (1), 59-83.

Avilés, J. M. y Elices, J. A. (2007). Insebull. Instrumentos para la evaluación del bullying. Madrid: CEPE.

Avilés, J. M., Irurtia, M. J., García-López, L. J. y Caballo, V. E. (2011). El maltrato entre iguales: "bullying". Behavioral Psychology/Psicologia Conductual, 19, 57-90.

Avilés, J. M. y Monjas, I. (2005). Estudio de la incidencia de la intimidación y el maltrato entre iguales en la educación secundaria obligatoria mediante el cuestionario CIMEI (Avilés, 1999) -Cuestionario sobre Intimidación y Maltrato Entre Iguales-. Anales de Psicología, 21 (1), 27-41.

Baldry, A. C. y Farrington, D. P. (2004). Evaluation of an intervention program for the reduction of bullying and victimization in school. Aggressive Behavior, 30, 1-15.

Bentler, P.M. (1977). Factor simplicity index and transformations. Psychometrika, 59, 567-579.

Bjorkqvist, K., Lagerspetz, K. y Kaukianen, A. (1992). Do girls manipulate and boys fight? Developmental trends in regard to direct and indirect aggression. Aggressive Behavior, 18, 117-127.

Buss, A. H. y Perry, M. (1992). The Aggression Questionnaire. Journal of Personality and Social Psychology, 63 (3), 452-459.

Caballo, V. E., Arias, B., Calderero, M., Salazar, I. C. e Irurtia, M. J. (2011). Acoso escolar y ansiedad social en niños (I): análisis de su relación y desarrollo de nuevos instrumentos de evaluación. Behavioral Psychology/Psicología Conductual, 19, 501-609.

Caballo, V. E., Calderero, M., Arias, B., Salazar, I. C. e Irurtia, M. J. (2012). Desarrollo y validación de una nueva medida de autoinforme para evaluar el acoso escolar (BULLYING). Behavioral Psychology/Psicología Conductual, 20, 625-647.

Caballo, V. E., Calderero, M., Carrillo, G. B., Salazar, I. C. e Irurtia, M. J. (2011). Acoso escolar y ansiedad social en niños (II): una propuesta de intervención en formato lúdico. Behavioral Psychology/Psicología Conductual, 19, 611-626.

Calderero, M., Salazar, I. C. y Caballo, V. E. (2011). Una revisión de las relaciones entre el acoso escolar y la ansiedad social. Behavioral Psychology/Psicología Conductual, 19, 389-415.

Carney, A. G. y Merrell, K. W. (2001). Bullying in schools: Perspective on understanding and preventing an international problem. School Psychology International, 36, 457-477. el diseño de la investigación limita los resultados obtenidos a un momento específico, por lo que continuamos realizando investigaciones complementarias en las que se evalúe la fiabilidad test-retest en intervalos temporales amplios para identificar si las puntuaciones se mantienen relativamente estables a lo largo del tiempo, con el fin de contribuir de manera precisa y efectiva a la evaluación de la violencia escolar infantil.

Agradecimientos.- Este trabajo de investigación ha recibido una ayuda a la Investigación del Instituto Alicantino de Cultura Juan Gil-Albert, organismo autónomo de la Diputación de Alicante, en su convocatoria 2011.

Caruana, A. (2005). Programa de Educación Emocional para la Prevención de la Violencia, $2^{\circ}$ ciclo de ESO. Valencia: Conselleria de Cultura, Educació i Esport. Generalitat Valenciana.

Caruana, A. (2007). Programa de Educación Emocional para la Prevención de la Violencia, $1^{\circ}$ ciclo de ESO. Valencia: Conselleria de Cultura, Educació i Esport. Generalitat Valenciana.

Caruana, A. (2011). Cultivando emociones: educación emocional de 3 a 8 años. Valencia: Conselleria d'Educació. Generalitat Valenciana.

Cattell, R. B. (1966). The scree test for the number of factors. Multivariate Behavioral Research, 1, 245-276.

Cerezo, F. (2006). Análisis comparativo de variables socioafectivas diferenciales entre los implicados en el bullying. Estudio de un caso de víctimaprovocador. Anuario de Psicología Clinica y de la Salud, 2, 27-34.

Cerezo, F. (2009). La violencia en las aulas. Análisis y propuestas de intervención. Madrid: Pirámide.

Cerezo, F. y Ato, M. (2010). Social status, gender, classroom climate and bullying among adolescents pupils. Anales de Psicologia, 26, 137-144.

Colell, J. y Escudé, C. (2006). El acoso escolar: un enfoque psicopatológico. Anuario de Psicología Clínica y de la Salud, 2, 9-14.

Craig, W. M., Pepler, D. J. y Atlas, R. (2000). Observations of bullying on the playground and in the classroom. International Journal of School Psychol ogy, 21, 22-36.

Crick, N. R., Ostrov, J. M., Burr, J. E., Cullerton-Sen, C., Jansen-Yeh, E. y Ralston, P. (2006). A longitudinal study of relational and physical aggression in preschool. Applied Developmental Psychology, 27, 254-268.

Defensor del Pueblo (1999). Violencia escolar: el maltrato entre iguales en la Educación Secundaria Obligatoria. Madrid: Publicaciones de la Oficina del Defensor del Pueblo.

Defensor del Pueblo (2006). Violencia Escolar: El maltrato entre Iguales en la Educación Obligatoria Secundaria 1999-2006. Madrid: Publicaciones de la Oficina del Defensor del Pueblo.

Defensor del Menor de la Comunidad de Madrid (2006). Convivencia, conflictos y educación en los centros escolares de la Comunidad de Madrid. Madrid: Publicaciones de la Oficina del Defensor del Menor.

Díaz-Aguado, M. J., Martínez, R. y Martín, G. (2004). Prevención de la violencia y lucha contra la exclusión desde la adolescencia. Madrid: Ministerio de Trabajo y Asuntos Sociales, INJUVE.

Díaz-Aguado, M. J. (2008). Estudio estatal sobre la convivencia escolar en la Educación Secundaria Obligatoria. Unidad de Psicología Preventiva de la UCM.

Dodge, K. A. y Coie, J. D. (1987). Social-information-processing factors in reactive and proactive aggression in children's peer groups. Journal of Personality and Social Psychology, 53 (6), 1146-1158.

Fernández, I. (1999). Prevención de la violencia y resolución de conflictos. El clima escolar como factor de calidad. Madrid: Narcea.

Fernández-Baena, F. J., Trianes, V., Morena-Fernández, M., EscobarEspejo, M., Infante-Cañete, L. y Blanca-Mena, M. (2011). Propiedades psicométricas de un cuestionario para la evaluación de la violencia cotidiana entre iguales en el contexto escolar. Anales de Psicología, 27, 102108. 
Galen, B. R. y Underwood, M. K. (1997). A developmental investigation of social aggression among children. Developmental Psychology, 33 (4), 589600 .

Garaigordobil, M. y Oñederra, J. A. (2010). La violencia entre iguales. Revisión teórica y estrategias de intervención. Madrid: Pirámide.

Garaigordobil, M. y Oñederra, J. A. (2011). Empatía y resolución de conflictos durante la infancia y la adolescencia. Revista Latinoamericana de Psicología, 43 (2), 255-266.

Hayton, J. C., Allen, D. G. y Scarpello, V. (2004). Factor retention decisions in exploratory factor analysis: A tutorial on parallel analysis. Organizational Research Methods, 7, 191-205.

Hoyle, R. H. y Duvall, J. L. (2004). Determining the number of factors in exploratory and confirmatory factor analysis. En D. Kaplan (Ed.). The Sage Handbook of Quantitative Methodology for the Social Sciences. Thousand Oaks: Sage.

Horn, J. L. (1965). A rationale and test for the number of factors in factor analysis. Psychometrika, 30, 179-185.

Kochenderfer, B. J., y Ladd, G. W. (1996). Peer victimization: Cause or consequence of school adjustment? Child Development, 67, 1305-1317.

Lorenzo-Seva, U. y Ferrando, P. J. (2006). FACTOR: A computer program to fit the exploratory factor analysis model. Behavioral Research Methods, Instruments and Computers, 38 (1), 88-91.

Malti, T., Perren, S. y Buchmann, M. (2010). Children's peer victimization, empathy, and emotional symptoms. Child Psychiatry and Human Development, 41 (1), 98-113.

Menesini, E., Codecasa, E., Benelli, B. y Cowie, H. (2003). Enhancing children's responsibility to take action against bullying: evaluation of a befriending intervention in Italian middle schools. Aggressive Behavior, 29, 1 14

Monclús, A., Oliva, J., Sánchez, P., Gonzalo, V. y Barrigüete, M. (2004). Bases para el análisis y diagnóstico de los conflictos escolares. Granada: Grupo Editorial Universitario.

Monks, C. P., Smith, P. K. y Swettenham, J. (2005). The Psychological Correlates of Peer Victimisation in Preschool: Social Cognitive Skills, Executive Function and Attachment Profiles. Aggressive Behavior, 31, 571588.

Monks, C. P., Palermiti, A., Ortega, R. y Costabile, A. (2011). A crossnational comparation of aggressors, victims and defenders in preschools in England, Spain and Italy. The Spanish Journal of Psychology, 14 (1), 133-144.

Morgan, D. L. (1998a). The Focus Group Guidebook. Focus Group Kit 1. Thousand Oaks, Ca: Sage.

Morgan, D. L. (1998b). Planning Focues Group. Focus Group Kit 2. Thousand Oaks, Ca: Sage.

Muñiz, J. y Fernández-Hermida, J. R. (2010). La opinión de los psicólogos españoles sobre el uso de los tests. Papeles del Psicólogo, 31 (1), 108-121.

Muthen, B. y Kaplan, D. (1992). A comparison of some methodologies for the factor analysis of non-normal Likert variables: A note on the size of the model. British Journal of Mathematical and Statistical Psychology, 45, 1930 .

Nunnally, J. y Bernstein, Y. (1995). Teoría psicométrica. México: McGrawHill.

Olweus, D. (2006). Conductas de acoso y amenaza entre escolares ( $3^{a}$ Ed.). Madrid: Ed. Morata.

Olweus, D. (2010). Understanding and researching bullying: Some critical issues. In S. R. Jimerson, S. M. Swearer y D. L. Espelage (Eds.), The handbook of school bullying: An international perspective (pp. 9-33). New York: Routledge.

O'Moore, A. M. y Minton, S. J. (2005). Evaluation of the effectiveness of an anti-bullying programme in primary schools. Aggressive Behavior, 31, 609622.

Ortega, R., Del Rey, R. y Mora-Merchán, J. (2001). Violencia entre escolares: conceptos y etiquetas verbales que definen el fenómeno del maltrato entre iguales. Revista Interuniversitaria de Formación del Profesorado, 41, 95113.
Ortega, R. y Monks, C. (2005). Agresividad injustificada entre preescolares. Psicothema, 17 (3), 453-458.

Ortega, R., Romera, E., Mérida, R. y Monks, C. (2009). Actividad e interacción entre iguales: explorando el mapping como instrumento de observación en aulas de Educación Infantil. Infancia y Aprendizaje, 32 (3), 405420.

Ortega, R., Sánchez, V. y Menesini, E. (2002). Bullying and moral disengagement: a cross-national comparison. Psicothema, 14, 37-49

Ostrov, J. M., Crick, N. R., y Stauffacher, K. (2006). Relational aggression in sibling and peer relationships during early childhood. Applied Developmental Psychology, 27, 241-253.

Peralta, J., Sánchez, M. D., Trianes, M. V. y De la Fuente, J. (2003). Estudio de la validez interna y externa de un cuestionario sobre conductas problemáticas para la convivencia según el profesor. Psicología, Saúde y Doencas, 4 (1), 83-96.

Perren, S. y Alsaker, F. D. (2006). Social behavior and peer relationship of victims, bully-victims and bullies in kindergarten. The Journal of Child Psychology and Psychiatry and Allied Disciplines, 47 (1), 165-176.

Perren, S. y Alsaker, F. D. (2009). Depressive symptoms from kindergarten to early school age: longitudinal associations with social skills deficits and peer victimization. Child and Adolescent Psychiatry and Mental Health, 3, 28.

Piñero-Ruíz, E. (2010). Características de las relaciones familiares y escolares y roles en la dinámica bullying en estudiantes de Educación Secundaria Obligatoria. Tesis doctoral. Universidad de Murcia.

Piñuel, I. y Oñate, A. (2005). Informe Cisneros VII: Violencia y acoso escolar en alumnos de Primaria, ESO y Bachiller. Instituto de Innovación Educativa y Desarrollo Directivo (IEDDI)

Piñuel, I. y Oñate, A. (2006). AVE. Acoso y violencia escolar. Madrid: TEA.

Raine, A., Dodge, K., Loeber, R., Gatzke-Kopp, L., Lynam, D., Reynolds, C., Stouthamer-Loeber, M., y Liu, J. (2006). The Reactive-Proactive Aggression Questionnaire: Differential correlates of reactive and proactive aggression in adolescent boys. Aggressive Behavior, 32 (2), 159-171.

Romera, E. M. y Ortega, R. (2006). La agresividad injustificada en edad infantil: qué es y cómo abordarla. Aula Infantil, 32, 42-46.

Ruiz, M. A., Pardo, A. y San Martín, R. (2010). Modelos de ecuaciones estructurales. Papeles del Psicólogo, 31 (1), 34-45.

Sánchez-Jiménez, V., Ortega, R. y Menesini, E. (2012). La competencia emocional de agresores y víctimas de "bulling". Anales de Psicología, 28, 71-82.

Sánchez-Lacasa, C. y Cerezo, F. (2010). Variables personales y sociales relacionadas con la dinámica bullying en escolares de Educación Primaria. Electronic Journal of Research in Educational Psychology, 8 (22), 1015-1032.

Sanmartín, J. (2004). El laberinto de la violencia. Causas, tipos y efectos. Barcelona: Ariel.

Serrano, A. (2006). Acoso y violencia en la escuela: cómo detectar, prevenir y resolver el bullying. Ariel: Centro Reina Sofía para el Estudio de la Violencia.

Vlachou, M., Andreou, E., Botsoglou, K. y Didaskalao, E. (2011). Bully/Victim Problems Among Preschool Children: a Review of Current Research Evidence. Educational Psychology Review 23, 329-358.

Vieira, M., Fernández, I. y Quevedo, G. (1998). Violence, Bullying and Counselling in the Iberian Península. En Roland, E. y Munthe, E. (Ed.), Bullying: An International Perspective (pp. 35-52) London: David Fulton Publishers.

Wolke, D., Woods, S., Schulz, H. y Stanford, K. (2001). Bullying and victimisation of primary school children in England and Germany: prevalence and school factors. British Journal of Psychology, 92, 673-696.

Zabalza, M.A. (1999). A convivencia nos centros escolares de Galicia. Santiago de Compostela: Instituto de Ciencias de la Educación-Consello Escolar de Galicia.

(Artículo recibido: 19-9-2012; revisado: 7-3-2013; aceptado: 8-5-2013) 
